\title{
The Influence of Aging on the Relationship between Retrobulbar Blood Flow and Retinal Microcirculation in Glaucoma Patients
}

Kendall M. Burgett, BS ${ }^{1}$, Brent Siesky, $\mathrm{PhD}^{2}$, Alon Harris, MS, FARVO, $\mathrm{PhD}^{2}$ ${ }^{1}$ Indiana University School of Medicine, ${ }^{2}$ Indiana University School of Medicine, Department of Ophthalmology, Indianapolis, Indiana, USA.

Background and Hypothesis: Glaucoma is a multifactorial disease; therefore, different patient populations may be more susceptible to developing glaucoma due to distinct factors. The hypothesis of this study was that the relationship between retrobulbar blood flow velocity and the amount of avascular area in the peripapillary retina in glaucoma patients differs based on patient age.

Project Methods: Data from 18 glaucoma patients aged $\geq 70$ years old $(75.9 \pm 5.4)$ and 24 glaucoma patients aged $<70(60.5 \pm 8.6)$ were used in this analysis. Peak systolic and end diastolic velocities (PSV/EDV) of nasal and temporal posterior ciliary arteries (N/TPCA) were measured using color Doppler imaging (CDI) in order to assess retrobulbar blood flow velocities. Heidelberg Retinal Flowmetry (HRF) was used to quantify the amount of superior avascular area (SAA) and inferior avascular area (IAA) of the peripapillary retina.

Results: In patients $\geq 70$ years of age, a negative correlation was detected between retrobulbar blood flow velocity and IAA ( $r=-0.45$ for NPCA-PSV, $r=-0.36$ for NPCA-EDV, $r=-0.35$ for TPCA-PSV, $r=-0.45$ for TPCA-EDV). This is in contrast to the positive correlation seen in patients $<70$ years of age $(r=0.45$ for NPCA-PSV, $r=0.39$ for NPCA-EDV, $r=0.55$ for TPCA-PSV, $r=0.69$ for TPCAEDV). Correlation coefficients between the two age groups were significantly different $(p<0.01, p<0.05, p<0.01$, and $p<0.0001$ respectively).

Conclusion and Potential Impact: The relationship between blood vessels supplying the eye and retinal microcirculation is significantly different based on patient age. This suggests that different age populations may have distinct risk profiles for glaucoma. A more complete understanding of population dependent risk factor profiles may assist glaucoma screening and early detection. 